\title{
Direct measurement of the magnetic penetration depth by magnetic force microscopy
}

\author{
Jeehoon Kim, ${ }^{1, \text { * }}$ L. Civale,${ }^{1}$ E. Nazaretski, ${ }^{2}$ N. Haberkorn, ${ }^{1}$ F. Ronning, ${ }^{1}$ A. \\ S. Sefat ${ }^{3}$ T. Tajima, ${ }^{1}$ B. H. Moeckly ${ }^{4}$ J. D. Thompson, ${ }^{1}$ and R. Movshovich ${ }^{1}$ \\ ${ }^{1}$ Los Alamos National Laboratory, Los Alamos, NM 87545 \\ ${ }^{2}$ Brookhaven National Laboratory, Upton, NY 11973 \\ ${ }^{3}$ Oak Ridge National Laboratory, Oak Ridge, Tennessee 37831 \\ ${ }^{4}$ Superconductor Technologies Inc., Santa Barbara, CA 93111
}

(Dated: November 3, 2018)

\begin{abstract}
We present an experimental approach using magnetic force microscopy for measurements of the absolute value of the magnetic penetration depth $\lambda$ in superconductors. $\lambda$ is obtained in a simple and robust way without introducing any tip modeling procedure via direct comparison of the Meissner response curves for a material of interest to those measured on a reference sample. Using a wellcharacterized $\mathrm{Nb}$ film as a reference, we determine the absolute value of $\lambda$ in a $\mathrm{Ba}\left(\mathrm{Fe}_{0.92} \mathrm{Co}_{0.08}\right)_{2} \mathrm{As}_{2}$ single crystal and $\mathrm{a} \mathrm{MB}_{2}$ thin film through a comparative experiment. Our apparatus features simultaneous loading of multiple samples, and allows straightforward measurement of the absolute value of $\lambda$ in superconducting thin film or single crystal samples.
\end{abstract}

\section{INTRODUCTION}

The superconducting coherence length $(\xi)$, magnetic penetration depth $(\lambda)$, and their anisotropy are fundamental parameters that characterize superconducting materials,$\underline{\underline{1}}-\mathbf{A}$ A number of important properties, such as superconducting critical fields and superconducting fluctuations that affect vortex dynamics, can be obtained if the parameters above are known. ${ }^{1,6}$ The value of $\xi$, which depends on the Fermi velocity and the condensation energy of the superconducting state, can be estimated from the upper critical field $\left(H_{c 2}\right)$ using the Ginzburg-Landau theory. $\lambda$ is related to the density of superconducting electrons, $\frac{1}{-1}$ and, in contrast to $\xi$, precise determination of its absolute value is notoriously difficult 1,2 due to demagnetizing effects, topography-related surface barrier, and inhomogeneity of the sample. Typically $\lambda$ is calculated by indirect methods. Several experimental techniques such as tunnel diode oscillator (TDO), $\underset{7}{t}$ temperature dependence of the flux explusion in the Meissner state $^{-}$or of the reversible magnetization in the mixed state using superconducting quantum interference device


impedance, 11 infrared reflectivity, 12 muon spin resonance $(\mu \mathrm{SR}), 13,14$ nuclear magnetic resonance (NMR), 15 and magnetic force microscopy $(\mathrm{MFM})^{16-19}$ have been employed for measurements of $\lambda$ in thin films and bulk samples. Each of these methods has its own advantages as well as limitations, and some of them require simulations with multiple fit parameters. For example, TDO measurements depend on the quality and thickness of an $\mathrm{Al}$ film deposited on top of a superconductor, which may not be fully reproducible yielding errors in the obtained values of $\lambda$. The temperature dependent Meissner response method is only sensitive for thin films with magnetic filed parallel to the surface. The reversible magnetization method works only in clean samples or materials having an extended vortex liquid phase. Mutual inductance and $\mu \mathrm{SR}$ techniques are limited to thin films and bulk samples, respectively. $\mu$ SR measures the second moment of the magnetic field distribution around vortex, consequently details of the vortex structure and the muon's location affect experimental accuracy. Infrared reflectivity allows measurement of the anisotropy $\lambda$ by polarization of an incident light.

MFM has been widely used for studies of superconductors, particularly for imaging and manipulation of vortices in superconducting thin films and single crystals ${ }^{20-24}$ Recently, MFM was also used as a local probe of the magnetic penetration depth, $17-19$ where the values of $\lambda$ were extracted by fitting either an MFM signal from a single vortex ${ }^{17}$ (note, this method doesn't work for systems with large values of the magneic penetration $\operatorname{depth}^{25}$ ) or a Meissner response ${ }^{18,19}$ These methods require a thorough characterization of the probe tip and a well-defined simulation model (dipole-monopole, monopole-monopole, etc.) describing interactions between the MFM tip and a superconducting sample. However, modeling procedures with multiple fitting parameters introduce uncertainties in the resulting $\lambda$ values. In spite of technical difficulties MFM has certain advantages e.g. localization of measured $\lambda$ values providing a route to explore anisotropy of $\lambda$ throughout the sample. In our approach, we obtain local values of $\lambda$ by directly comparing the Meissner curves for the sample under investigation with those obtained for a reference sample with a well-known $\lambda$; we emphasize that the measurements are done using the same MFM tip during the same cooldown. We observed strong dependence of the Meissner response curves on the shape of the MFM tip. When the tip crashes or even slightly touches the sample surface, Meissner response curves can change significantly. During our experimental procedure we verify that the MFM tip does not change its properties by comparing the Meissner curve from a $\mathrm{Nb}$ reference sample before and after the measurement. In this paper we demonstrate the validity of our method by determining $\lambda$ in a $\mathrm{Ba}\left(\mathrm{Fe}_{0.92} \mathrm{Co}_{0.08}\right)_{2} \mathrm{As}_{2}$ single crystal and a $\mathrm{MgB}_{2}$ film. Our results demonstrate that the same procedure can be 
used in any single crystal or thin film superconducting samples with a thickness greater than $\lambda$ (for thinner samples $\lambda$ can be corrected in a straightforward manner ${ }^{26,27}$ )

\section{EXPERIMENT}

The measurements described in this paper were performed in a home-built low-temperature MFM apparatus. ${ }^{28}$ We have developed an additional capability of mounting multiple samples (including a reference sample), as shown in Fig. 1(a), for acquiring a complete set of MFM data for each of the samples within a single cool-down. The absolute value of $\lambda$ is obtained by a simple comparison of the Meissner curves for the $\mathrm{Nb}$ reference to those obtained in the sample of interest. The Meissner response curve is first measured in a homogeneous reference sample ( $\mathrm{Nb}$ film) as a function of the tip-sample separation. Then the cantilever is moved over the sample of interest, and its Meissner response curve is obtained. Direct comparison of these curves (comparative experiments) yields the absolute value of $\lambda$ in a sample under investigation. The value of $\lambda$ in the reference sample ( $\mathrm{Nb}$ film) was verified by a different MFM technique and a SQUID magnetometry measurement 17 The reference $\mathrm{Nb}$ thin film $\left(T_{c} \approx 8.8 \mathrm{~K}\right.$, where resistance drops to zero) has a thickness of $300 \mathrm{~nm}$ and was grown by an electron beam deposition. $\mathrm{A} \mathrm{Ba}\left(\mathrm{Fe}_{0.92} \mathrm{Co}_{0.08}\right)_{2} \mathrm{As}_{2}$ single crystal $\left(T_{c} \approx 22 \mathrm{~K}\right.$ from specific heat capacity measurements) was grown out of FeAs flux. ${ }^{29.30}$ The 500-nm thick $\mathrm{MgB}_{2}$ film $\left(T_{c} \approx 38.3 \mathrm{~K}\right.$, zero resistance temperature) was grown by reactive evaporation 31 The MFM measurements were performed using a high resolution Nanosensors cantilever ${ }^{32}$ that was polarized along the tip axis in a $3 \mathrm{~T}$ field of a superconducting magnet. The superconducting samples are zero field-cooled for the Meissner experiment and are field-cooled in a field of few Oersted, applied perpendicular to the film surface and parallel to the probe tip, for imaging of vortices. All samples are electrically grounded to eliminate a possible electric force contribution from a stray charge on the sample to the magnetic Meissner force.

\section{RESULTS AND DISCUSSION}

\section{A. MFM measurement in the $\mathrm{Nb}$ film}

As the magnetic tip approaches a superconducting sample, it experiences a Meissner force (Meissner response) induced by the shielding currents in the sample that screen the magnetic field of the tip. The experimental procedure is as follows: First the probe tip is brought close to the reference sample [Nb film, position 1 in panel (a) of Fig. 1 and the Meissner response is recorded as a function of the tip-sample separation at a certain temperature $T$. There should be no vortices present in a large field of view of the sample, i.e., the sample should remain (a)

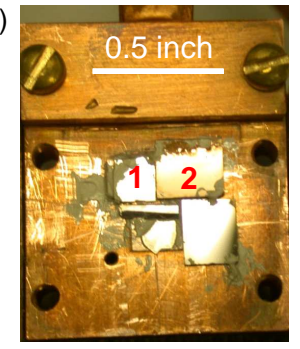

(b)

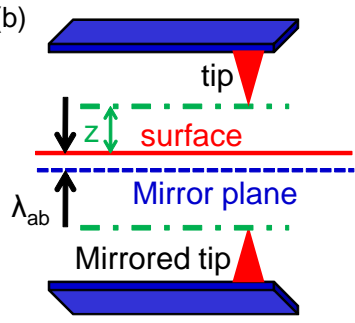

FIG. 1: (Color online) (a) Sample holder with multiple samples. The $\mathrm{Nb}$ thin film $(300 \mathrm{~nm})$ and the $\mathrm{MgB}_{2}$ thin film (500 nm) samples are labeled as 1 and 2, respectively. (b) Schematic illustration of the Meissner experiment. The Meissner response force between the probe tip and the sample can be regarded as the force between the real tip and image tip at $2(z+\lambda)$, when $z \gg \lambda$. 18

in a pure Meissner state. Figure 2(a) shows the Meissner state with no vortices present in the $25 \mu \mathrm{m} \times 25 \mu \mathrm{m}$ field of view after a small compensation field was applied above $T_{c}$ of $\mathrm{Nb}$ to compensate the remnant stray field of the superconducting magnet.

Figure 2(b) shows several Meissner response curves for the $\mathrm{Nb}$ film reference sample between $5.5 \mathrm{~K}$ and $8 \mathrm{~K}$. The Meissner response force is a function of $\lambda$ and the tip-sample separation $z, F_{M}=F(z+\lambda), 18,33$ For a given temperature $T, F_{M}[z+\lambda(T)]=F_{M}[z+\lambda(4 \mathrm{~K})+\delta \lambda]$, where $\delta \lambda=\lambda(T)-\lambda(4 \mathrm{~K})$. Therefore, to determine the $\lambda$ value at a particular temperature $T, F_{M}[z+\lambda(T)]$ is shifted along the $z$ axis to coincide with $F_{M}[z+\lambda(4 \mathrm{~K})]$, a reference curve measured at $4 \mathrm{~K}$, and the value of the shift yields the value of $\delta \lambda(T)$.

The Meissner response curve taken at $8 \mathrm{~K}$ shows a behavior very different from the data taken at lower temperatures. Figure 2(c) shows the MFM image acquired at $4 \mathrm{~K}$ after the Meissner measurement was performed at 8 K. The scan areas in Fig. 2(a) and Fig. 2(c) are the same. The vortices seen in Fig. 2(c) were generated by the field of the probe tip. They cause magnetic field leakage and weaken the Meissner response (see Fig. 2(b), $8 \mathrm{~K}$ curve). Such a behavior is observed when $\lambda$ is comparable to the film thickness, and the magnetic field from the tip can not be fully screened, ${ }^{26,27}$ Special care must therefore be taken with the Meissner technique in the vicinity of $T_{c}$ in thin films since increasing $\lambda$ may take the sample out of the pure Meissner state.

To verify the $4 \mathrm{~K}$ value of $\lambda(4 \mathrm{~K})=110 \pm 10 \mathrm{~nm}$ for the $\mathrm{Nb}$ reference, measured previously using a different MFM technique, $\stackrel{17}{ }$ we performed measurements of $\lambda$ using the SQUID magnetometry. The $\mathrm{Nb}$ reference sample used in MFM measurements (film $\mathrm{L}=3.2 \mathrm{~mm}$ $\times \mathrm{W}=4.2 \mathrm{~mm}$ ) was oriented carefully with $\mathbf{H}$ parallel to the surface along the side L, using a home-built sample holder. By measuring the transverse component $m_{\perp}$ of the magnetic moment $\mathbf{m}$ in the Meissner state 

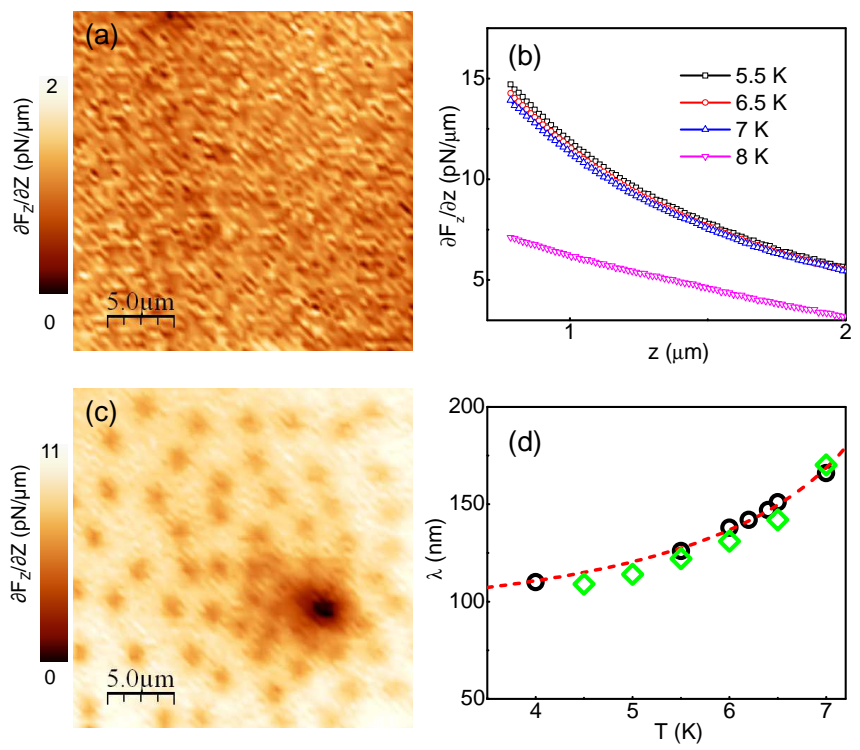

FIG. 2: (Color online) (a) MFM image of the $\mathrm{Nb}$ sample at $T=4 \mathrm{~K}$; no vortices are present in a $25 \mu \mathrm{m} \times 25 \mu \mathrm{m}$ field of view. (b) Meissner response curves taken over the scan area shown in (a). (c) The MFM image taken with the same field of view as in (a) after the Meissner response experiment at $8 \mathrm{~K}$; vortices are nucleated by the magnetic field of the MFM tip. (d) Temperature dependence of the penetration depth $\lambda(T)$ in $\mathrm{Nb}$ obtained by overlaying Meissner curves on top of the $4 \mathrm{~K}$ reference curve (black circles). Green squares represent SQUID data; the red-dashed curve is a fit to the BCS model.

(which should be zero in the case of perfect alignment) we confirmed that the miss-orientation between $\mathbf{H}$ and the film surface was $\phi_{\text {mis }} \sim 0.2^{\circ}$. In this configuration, in the Meissner state the component of $\mathbf{m}$ parallel to $\mathbf{H}$ is $m_{\|}=(H / 4 \pi) \times L W d_{e f f}$, where the effective thickness $d_{\text {eff }}(T)=d-2 \lambda(T) \tanh (d / 2 \lambda(T))$ is smaller than the geometrical thickness due to the field penetration from both surfaces $\stackrel{1,2}{=}$ We measured $m_{\|}$versus $H$ at several $T$, and from the slopes $d m_{\|} / d H$ we extracted $\lambda(T)$. The main source of error in this method is the spurious contribution to $m_{\|}$due to the projection of the Meissner signal arising from the transverse field component, $\sim m_{\perp} \phi_{m i s}$, which is $\sim 10 \%$ of $m_{\|}$, thus introducing an error of $\sim 5 \%$. However, it does not distort the functional dependence of $\lambda(T)$. The absolute values of $\lambda(T)$ from these SQUID measurements are marked with green squares in Fig. 2(d) and agree well with both the MFM data (black circles) and the isotropic single-gap BCS model (red-dashed curve).

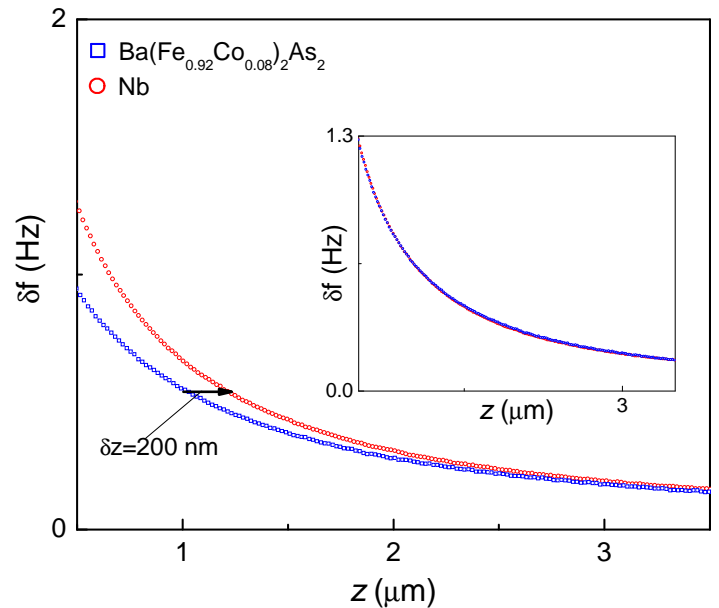

FIG. 3: (Color online) Meissner response curves obtained from (a) the $\mathrm{Nb}$ reference, (b) a $\mathrm{Ba}\left(\mathrm{Fe}_{0.92} \mathrm{Co}_{0.08}\right)_{2} \mathrm{As}_{2}$ single crystal at $4 \mathrm{~K}$. Different slopes of the Meissner curves obtained from each sample indicate a systematic change of $\lambda$. Inset: The Meissner curve of $\mathrm{Ba}\left(\mathrm{Fe}_{0.92} \mathrm{Co}_{0.08}\right)_{2} \mathrm{As}_{2}$ is shifted by $z=200 \mathrm{~nm}$ along the $z$ axis to overlay the Meissner curve of the $\mathrm{Nb}$ reference sample. The difference of the penetration depths $\Delta \lambda=200 \mathrm{~nm}$ can be obtained from the value of the shift along the $z$ axis.

\section{B. Measurements of the absolute values of $\lambda$ in a $\mathrm{Ba}\left(\mathrm{Fe}_{0.92} \mathbf{C o}_{0.08}\right)_{2} \mathbf{A s}_{2}$ single crystal}

In recent years iron-based pnictide superconductors have drawn a great deal of attention since these systems exhibit superconducting properties which are intermediate to conventional BCS superconductors and high- $\mathrm{T}_{c}$ cuprates. Magnetic penetration depth of a $\mathrm{Ba}\left(\mathrm{Fe}_{1-x} \mathrm{Co}_{x}\right)_{2} \mathrm{As}_{2}$ system (BFCA), the so-called 122 family, was investigated using variety of techniques including TDO $\stackrel{34}{\underline{34}}, \stackrel{35}{\underline{3}}$ and MFM $\stackrel{19}{=}$ The Stanford group utilized an MFM technique and reported absolute values of $\lambda$ in this system as a function of doping level. The authors used fitting algorithms to approximate the tip magnetization and calculate the values of $\lambda \underline{\underline{19}} \mathrm{We}$ applied our direct (comparative) technique to measurements of $\lambda$ in the very same system $\left[\mathrm{Ba}\left(\mathrm{Fe}_{1-x} \mathrm{Co}_{x}\right)_{2} \mathrm{As}_{2}\right.$ $(\mathrm{x}=0.08)]$ to demonstrate the validity of our approach. Figure 3 shows Meissner curves as a function of the tipsample separation $z$ obtained from BFCA (blue squares) and a $\mathrm{Nb}$ reference (red circles), respectively. The slow decay of a frequency shift in BFCA sample compared to that in the $\mathrm{Nb}$ reference indicates greater values of $\lambda$ in the BFCA sample. The expression for the Meissner response force in $\mathrm{Nb}$ (assuming monopole-monopole interaction between the tip and the sample) can be written as follows: $F_{\text {Meissner }}^{N b}=\frac{A \Phi_{0}}{\left(z+\lambda_{a b}^{N b}\right)^{3}}$, where $A$ is a prefactor that reflects the sensor's geometry and the magnetic moment, $\Phi_{0}$ is a single magnetic flux quantum, $z$ is the tip-sample separation, and $\lambda_{a b}^{N b}$ is the in-plane 

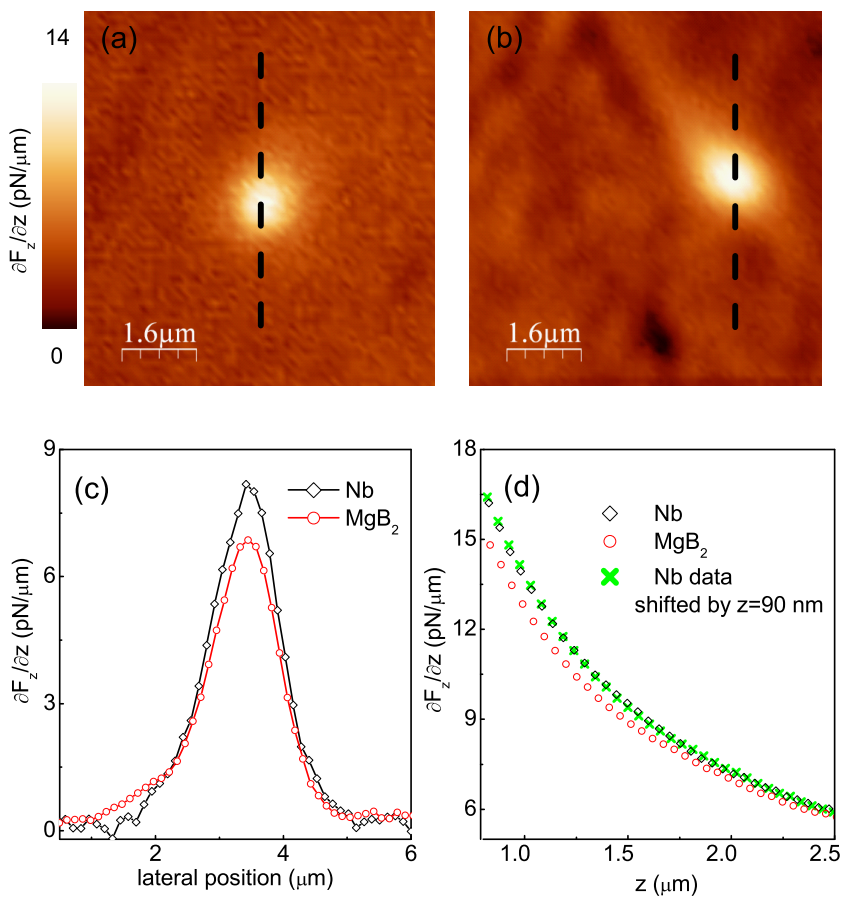

FIG. 4: (Color online) Single vortex images in (a) the $\mathrm{Nb}$ thin film and (b) the $\mathrm{MgB}_{2}$ thin film samples at $T=4 \mathrm{~K}$. Both images were taken with a tip-lift height of $400 \mathrm{~nm}$. (c) The single vortex profiles taken along the dotted lines in (a) and (b). (d) Meissner response curves taken at $4 \mathrm{~K}$ in Nb (black square) and $\mathrm{MgB}_{2}$ (red circle) with the same experimental condition. The green-crossed marks represent that the red circle $\left(\mathrm{MgB}_{2}\right)$, after shifted by $90 \mathrm{~nm}$ along the $z$ axis, is overlaid over the black square $(\mathrm{Nb})$ to show the validity of our approach.

magnetic penetration depth since the shielding current runs within the basal plane. The Meissner force in BFCA has the same functional form but different $\lambda^{B F C A}$ : $F_{\text {Meissner }}^{B F C A}=\frac{A \Phi_{0}}{\left(z+\lambda^{B F C A}\right)^{3}}$. Two Meissner curves become identical $\left(F_{\text {Meissner }}^{B F C A}=F_{\text {Meissner }}^{N b}\right)$ when the tip lift $z$ compensates for differences $\lambda^{B F C A}-\lambda^{N b}=\delta \lambda$. In other words, by shifting the Meissner curve for BFCA along the $z$ axis to overlay the Meissner curve measured for $\mathrm{Nb}$, the $\delta \lambda$ value can be extracted. By adding the shifted value $\delta z=\delta \lambda=200 \mathrm{~nm}$ to the $\lambda^{N b}$ (110 nm) one obtains $\lambda^{B F C A}=310 \pm 30 \mathrm{~nm}$. This value is close to the one reported previously 19 The inset in Fig. 3 demonstrates that two Meissner curves overlay each other very well after shifting the BFCA curve along the $z$ axis by 200 nm.

\section{Measurement of the absolute value of $\lambda$ in a $\mathrm{MgB}_{2}$ film}

We used the same approach to measure the absolute value of $\lambda$ in $\mathrm{a} \mathrm{MgB}_{2}$ thin film sample. The Meissner response curve taken at $4 \mathrm{~K}$ in the $\mathrm{Nb}$ was used as a reference Meissner curve and compared to the Meissner response curve measured in $\mathrm{MgB}_{2}$. The offset between these two curves, shown in Fig. 4 (d), yields the absolute value of $\lambda$ at $4 \mathrm{~K}$. The $\delta \lambda$ between the $\mathrm{Nb}$ and $\mathrm{MgB}_{2}$ curves at $4 \mathrm{~K}$ equals $90 \mathrm{~nm}$ yielding $\lambda(4 \mathrm{~K})=200 \pm 30$ $\mathrm{nm}$ in $\mathrm{MgB}_{2}$. Our experimental error is $10 \%-15 \%$, and depends on the magnitude of $\lambda$ and the system noise level. Absolute values of $\lambda$ in $\mathrm{MgB}_{2}$ measured with various techniques range from $40 \mathrm{~nm}$ to $210 \mathrm{~nm} \stackrel{36-42}{ }$ The large variation of $\lambda$ in $\mathrm{MgB}_{2}$ may be due to inclusion of impurities, such as $\mathrm{C}, \mathrm{N}$, and $\mathrm{Al}$, which replace either $\mathrm{Mg}$ or B and significantly affect the electronic structure of the system due to the two-band nature of $\mathrm{MgB}_{2}$. The origin of the impurities-induced large value of $\lambda$ and its temperature dependence obtained from the Meissner response curves in the $\mathrm{MgB}_{2}$ film taken at different temperatures (not shown) will be described elsewhere 43

We also imaged individual vortices in both $\mathrm{Nb}$ and $\mathrm{MgB}_{2}$. Direct comparison of the vortex profiles provides additional information on the magnitude of $\lambda$. The maximum force gradient at the center of a vortex, $\max (\partial f / \partial z)$ (MFM is sensitive to a force gradient), is proportional to $\left(z+\lambda_{a b}\right)^{-1 / 3}$ for a monopole-monopole model of the tip-


of $\lambda$ results in a smaller force gradient at the center of a vortex. Figures 4(a)-(b) show well-isolated vortices acquired in the $\mathrm{Nb}$ reference and the $\mathrm{MgB}_{2}$ sample at 4 $\mathrm{K}$. We estimate the magnetic field to be no more than 0.2 Oe based on the field calibration. Direct comparison of vortex profiles [see Fig. 4(c)], taken along the dotted lines in Figs. 4(a) and (b), shows that the force gradient in the $\mathrm{MgB}_{2}$ sample is smaller than that in the $\mathrm{Nb}$ sample. This indicates a larger value of $\lambda$ in our $\mathrm{MgB}_{2}$ film. It is worth noting that the relatively high tip-sample separation (400-nm tip-lift) and the tip geometry are responsible for the broadening of the vortex force profiles in both $\mathrm{Nb}$ and $\mathrm{MgB}_{2}$ samples in Fig. 4(c).

\section{CONCLUSION}

In conclusion, we have developed an experimental method and apparatus to determine the absolute value of the magnetic penetration depth $\lambda$ in superconducting samples by comparing their Meissner response curves to those acquired for a homogeneous $\mathrm{Nb}$ reference film. We used this method to obtain the absolute value of $\lambda(4$ $\mathrm{K})=310 \pm 30 \mathrm{~nm}$ in a $\mathrm{Ba}\left(\mathrm{Fe}_{0.92} \mathrm{Co}_{0.08}\right)_{2} \mathrm{As}_{2}$ single crystal, consistent with the value reported by the Stanford group. We also measured $\lambda(4 \mathrm{~K})=200 \pm 30 \mathrm{~nm}$ in a $\mathrm{MgB}_{2}$ film. The large $\lambda$ comes from the nature of the two band superconductivity, and from inclusion of impu- 
rities such as $\mathrm{C}$ and $\mathrm{N}$. Our MFM apparatus allows us to simultaneously load and investigate multiple samples (over ten samples can be studied at once, providing an opportunity to explore the complete phase diagram of a superconducting system), and most importantly to use the same cantilever tip for both $\mathrm{Nb}$ reference and the samples under investigation in a single cool-down. This capability enables in-situ calibration of the MFM tip on a known homogeneous $\mathrm{Nb}$ sample and does not introduce any additional uncertainties due to modeling of the tip geometry and the resulting tip field. The validity of our approach is established by comparing the MFM and SQUID magnetometer measurements of the temperature dependence of $\lambda$ in the $\mathrm{Nb}$ reference film. Our experimental approach opens the possibility of measuring the absolute value of $\lambda(T)$ in film and bulk superconducting samples.

The authors thank Y. Q. Wang for the RBS measurement. Work at LANL was supported by the US Department of Energy, Basic Energy Sciences, Division of Materials Sciences and Engineering (MFM, data analysis and manuscript preparation), and by T. Tajima 2010 DOE Early Career Award (SQUID measurements). Work at Brookhaven (data analysis and manuscript preparation) was supported by the US Department of Energy under Contract No. DE-AC02-98CH10886. BaFe ${ }_{2} \mathrm{As}_{2}$ samples were grown at Oak Ridge National Laboratory with the support of the Department of Energy, Basic Energy Sciences, Materials Sciences and Engineering Division. N.H. is member of CONICET (Argentina).
* Corresponding author: jeehoon@lanl.gov

1 M. Tinkham, Introduction to Superconductivity (McGrawHill Book Co., New York, 1996).

2 C. Poole, H. Farach, R. Creswick, and R. Prozorov, Superconductivity (Academic Press, Elsevier Ltd., Burlington, MA, 2007).

3 R. Prozorov, R. W. Giannetta, P. Fournier, and R. L. Greene, Phys. Rev. Lett. 85, 3700 (2000).

${ }^{4}$ I. Bonalde, Brian D. Yanoff, M. B. Salamon, D. J. Van Harlingen, E. M. E. Chia, Z. Q. Mao and Y. Maeno, Phys. Rev. Lett. 85, 4775 (2000).

${ }^{5}$ W. N. Hardy, D. A. Bonn, D. C. Morgan, R. Liang, and K. Zhang, Phys. Rev. Lett. 70, 3999 (1993).

${ }^{6}$ G. Blatter, M. V. Feigel'man, V. B. Geshkenbein, A. I. Larkin, and V. M. Vinokur, Rev. Mod. Phys. 66, 1125 (1994).

7 R. Prozorov, R. W. Giannetta, A. Carrington, P. Fournier, R. L. Greene, P. Guptasarma, D. G. Hinks, and A. R. Banks, Appl. Phys. Lett. 77, 4202 (2000).

${ }^{8}$ L. Civale and F. de la Cruz, Phys. Rev. B 36, 3560 (1987).

9 J. R. Thompson, D. K. Christen, H. A. Deeds, Y. C. Kim, J. Brynestad, S. T. Sekula, and J. Budai, Phys. Rev. B 41, 7293 (1990).

10 A. T. Fiory, A. F. Hebard, P. M. Mankiewich, and R. E. Howard, Appl. Phys. Lett. 52, 2165 (1988).

11 J. Mao, D. H. Wu, J. L. Peng, R. L. Greene, and S. M. Anlage, Phys. Rev. B 51, 3316 (1995).

12 D. N. Basov, R. Liang, D. A. Bonn, W. N. Hardy, B. Dabrowski, M. Quijada, D. B. Tanner, J. P. Rice, D. M. Ginsberg, and T. Timusk, Phys. Rev. Lett. 74, 598 (1995).

13 J. E. Sonier, J. H. Brewer, and R. F. Kiefl, Rev. Mod. Phys. 72, 769 (2000).

14 J. E. Sonier, R. F. Kiefl, J. H. Brewer, D. A. Bonn, J. F. Carolan, K. H. Chow, P. Dosanjh, W. N. Hardy, Ruixing Liang, W. A. MacFarlane, P. Mendels, G. D. Morris, T. M. Riseman, and J. W. Schneider, Phys. Rev. Lett. 72, 744 (1994).

15 B. Chen, P. Sengupta, W. P. Halperin, E. E. Sigmund, V. F. Mitrović, M. H. Lee, K. H. Kang, B. J. Mean, J. Y. Kim, and B. K. Cho, New J. Phys. 8274 (2006).

16 M. Roseman and P. Grtter, New J. Phys. 3, 24 (2001).

17 E. Nazaretski, J. P. Thibodaux, I. Vekhter, L. Civale, J.
D. Thompson, and R. Movshovich, Appl. Phys. Lett. 95, 262502 (2009).

${ }^{18}$ L. Luan, O. M. Auslaender, T. M. Lippman, C. W. Hicks, B. Kalisky, J. Chu, and K. A. Moler, Phys. Rev. B 81, 100501(R) (2010).

19 L. Luan, T. M. Lippman, C. W. Hicks, J. A. Bert, O. M. Auslaender, J.-H. Chu, J. G. Analytis, I. R. Fisher, and K. A. Moler, Phys. Rev. Lett. 106, 067001 (2011).

20 A. Moser, H. J. Hug, I. Parashikov, B. Stiefel, O. Fritz, H. Thomas, A. Baratoff, and H.-J. Güntherodt, Phys. Rev. Lett. 74, 1847 (1995).

21 A. Volodin, K. Temst, C. Haesendonck, and Y. Bruynseraede, Physica B 284, 815 (2000).

22 M. Roseman and P. Grütter, Appl. Surf. Sci. 188, 416 (2002).

23 O. M. Auslaender, L. Luan, E. W. J. Straver, J. E. Hoffman, N. C. Koshnick, E. Zeldov, D. A. Bonn, R. Liang, W. N. Hardy, and K. A. Moler, Nature Phys. 5, 35 (2009).

24 E. W. J. Straver, J. E. Hoffman, O. M. Auslaender, D. Rugar, and Kathryn A. Moler, Appl. Phys. Lett. 93, 172514 (2008).

25 J. Kim, F. Ronning, N. Haberkorn, L. Civale, E. Nazaretski, Ni Ni, R. J. Cava, J. D. Thompson, and R. Movshovich, Phys. Rev. B 85, 180504(R) (2012).

26 J. C. Wei, J. L. Chen, L. Hong, and T. J. Yang, Physica C 267, 345 (1996).

27 J. H. Xu, J. H. Miller Jr., and C. S. Ting, Phys. Rev. B 51, 424 (1995).

28 E. Nazaretski, K. S. Graham, J. D. Thompson, J. A. Wright, D. V. Pelekhov, P. C. Hammel, and R. Movshovich, Rev. Sci. Instrum. 80, 083074 (2009).

29 A. S. Sefat, R. Jin, M. A. McGuire, B. C. Sales, D. J. Singh, and D. Mandrus, Phys. Rev. Lett. 101, 117004 (2008).

${ }^{30}$ K. Gofryk, A. B. Vorontsov, I. Vekhter, A. S. Sefat, T. Imai, E. D. Bauer, J. D. Thompson, and F. Ronning, Phys. Rev. B 83, 064513 (2011).

31 B. H. Moeckly and W. S. Ruby, Supercond. Sci. Technol. 19, L21 (2006).

32 A SSS-QMFMR cantilever, Nanosensors, Inc.

33 L. Luan, O. M. Auslaender, N. Shapira, D. A. Bonn, R. Liang, W. N. Hardy, K. A. Moler, e-print arXiv:1103.6072 1 . 
${ }^{34}$ R. T. Gordon, C. Martin, H. Kim, N. Ni, M. A. Tanatar, J. Schmalian, I. I. Mazin, S. L. Bud'ko, P. C. Canfield, R. Prozorov Phys. Rev. B 79, 100506 (2009).

35 O. Ofer, J. C. Baglo, M. D. Hossain, R. F. Kiefl, W. N. Hardy, A. Thaler, H. Kim, M. A. Tanatar, P. C. Canfield, R. Prozorov, G. M. Luke, E. Morenzoni, H. Saadaoui, A. Suter, T. Prokscha, B. M. Wojek, and Z. Salman, Phys. Rev. B 85, 060506 (2012).

36 X. X. Xi. Rep. Prog. Phys. 71 (2008) 116501.

37 A. A. Golubov, A. Brinkman O. V. Dolgov, J. Kortus, and O. Jepsen, Phys. Rev. B 66, 054524 (2002), and references therein.

38 T. Dahm and D. J. Scalapino, Appl. Phys. Lett. 85, 4436 (2004).
39 X. K. Chen, M. J. Konstantinović, J. C. Irwin, D. D. Lawrie, and J. P. Franck, Phys. Rev. Lett. 87, 157002 (2001).

${ }^{40}$ K. H. Lee, K. H. Kang, B. J. Mean, M. H. Lee, and B. K. Cho, J. Magnet. Magnet. Mater. 272, 165 (2004).

41 F. Simon, A. Jánossy, T. Fehér, and F. Murányi, Phys. Rev. Lett. 87, 047002 (2001).

42 D. K. Finnemore, J. E. Ostenson, S. L. Bud'ko, G. Lapertot, and P. C. Canfield, Phys. Rev. Lett. 86, 2420 (2001).

43 J. Kim et al. (unpublished).

44 T. Shapoval, H. Stopfel, S. Haindl, J. Engelmann, D. S. Inosov, B. Holzapfel, V. Neu, and L. Schultz, Phys. Rev. B 83, 214517 (2011) 